\title{
Detection of a Rare Complication of Congenital Cystic Adenomatoid Malformation in a Recipient Twin With Twin-Twin Transfusion Syndrome Using Prenatal Magnetic Resonance Imaging
}

\author{
Yu-Peng Liu, ${ }^{1,2}$ Yang-Kai Fan, ${ }^{1,2}$ Yi-Lan Lin, ${ }^{1,2,}{ }^{*}$ Chih-Ping Chen, ${ }^{3,4}$ and Wen-Ko Su ${ }^{1,2}$ \\ ${ }^{1}$ Department of Radiology, Mackay Memorial Hospital, Hsinchu, Taiwan \\ ${ }^{2}$ Mackay Junior College of Medicine, Nursing and Management, Taipei, Taiwan \\ ${ }^{3}$ Department of Obsterics and Gyneco, Mackay Memorial Hospital, Taipei, Taiwan \\ ${ }^{4}$ Department of Medical Research, Mackay Memorial Hospital, Taipei, Taiwan \\ "Corresponding author: Yi-Lan Lin, Department of Radiology, Mackay Memorial Hospital, Hsinchu, Taiwan. Tel: +886-36119595, Fax: +886-225239437, E-mail: \\ a5338@ms7.mmh.org.tw
}

Received 2013 June 25; Revised 2013 December 11; Accepted 2014 April 15.

Keywords: Congenital Cystic Adenomatoid Malformation, Recipient, Twin-Twin Transfusion Syndrome, Magnetic Resonance Imaging

\section{Dear Editor,}

We would like to report a case of recipient twin with twin-twin transfusion syndrome (TTTS) that developed a very rare complication of congenital cystic adenomatoid malformation (CCAM) detected by magnetic resonance imaging (MRI) but not by ultrasonography (US). MRI was found to be more efficient than prenatal US for the detection of CCAM lesions.

A 30-year-old, gravida 3, para 2 woman who had developed TTTS at 24 weeks' gestation was referred to our hospital for evaluation of complications in the recipient twin owing to the death of the donor twin. The patient and her husband were healthy and denied any family history of genetic disorders or congenital malformations. The US findings of the recipient twin were normal (Figure 1), and no associated fetal anomalies, except polyhydramnios, were observed. Single-shot fast spin-echo sequence MRI was performed at the $25^{\text {th }}$ gestational week for further evaluation of fetal complications. Findings of the brain were normal, but several small wedge-shaped hyperintense lesions were observed along the periphery of both right and left lungs (Figure $2 \mathrm{~A}-\mathrm{C}$ ). The characteristics of these lesions were most consistent with CCAM. The parents decided to terminate the pregnancy. At 26 weeks of gestation, the fetus was delivered with a body weight of $698 \mathrm{~g}$, a normal karyotype of $46 \mathrm{XX}$ and the diagnosis of CCAM was confirmed by subsequent autopsy.

Recipient twin in TTTS may be complicated by brain or heart damage, and rarely CCAM $(1,2)$. The use of US in cases of TTTS has been well documented, and US is the primary imaging modality in these pregnancies (3). This case highlights the contribution of MRI to the prenatal diagnosis of

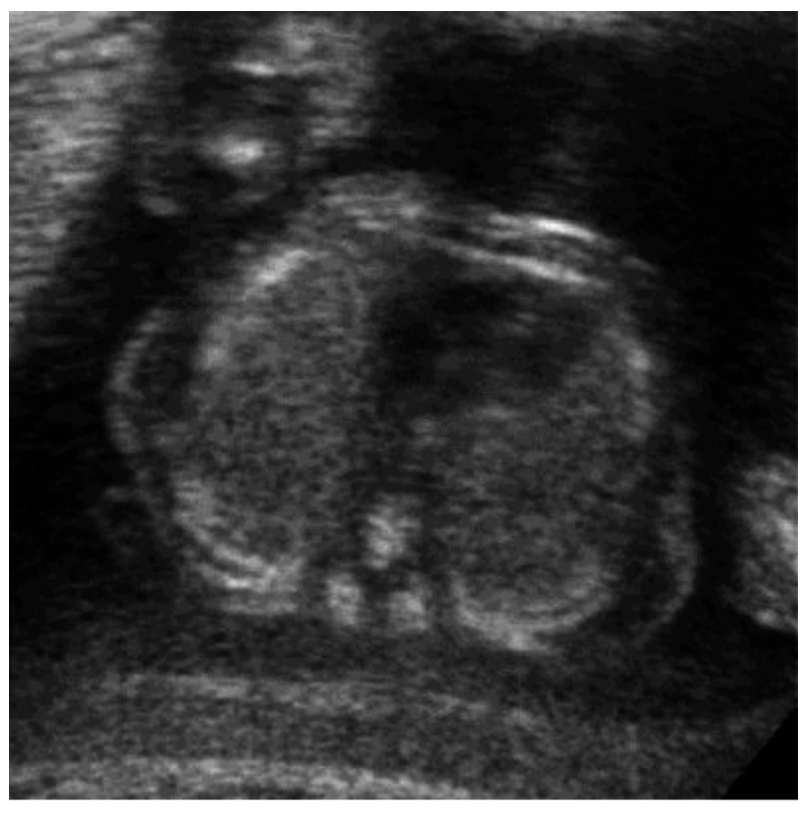

Figure 1. A gravida 3, para 2 pregnant woman with twin-twin transfusion syndrome at 24 weeks' gestation and death of one of the fetuses. Axial ultrasonography of the chest shows no abnormality.

lung lesions that cannot be detected by US. Therefore, MRI is a more valuable tool for the evaluation of fetal complications such as CCAM in the case of TTTS and it has benefits over US $(1,2)$. Six cases of CCAM in the recipient twin with TTTS have been reported in the literature that have been detected by prenatal MRI while they had normal US findings $(1,2)$. On MRI, these lesions may appear as minor 

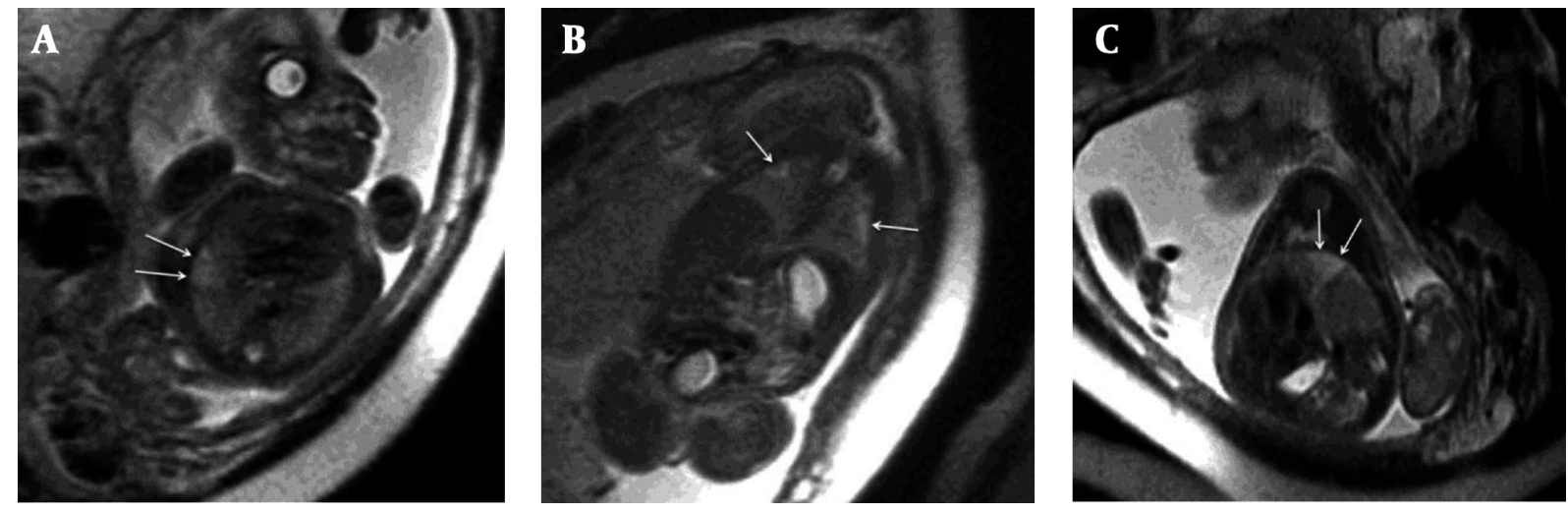

Figure 2. MRI of the chest of the fetus at 25 weeks gestation. A, Axial; B, Coronal; C, Sagittal views.

wedge-shaped hyperintense lesions along the lung periphery (1), or, as in our case, appear as severe, diffuse, small ill-defined hyperintense lesions on both lungs (2). TTTS is a complication of monochorionic/diamniotic twin pregnancies. It is characterized by an imbalance in blood flow through placental anastomoses causing potentially significant morbidity and mortality in both twins $(1,3)$. CCAM is characterized by adenomatoid proliferation of the cysts in the lung that resemble bronchioles. The pathogenesis of CCAM remains unknown. Increased cell proliferation, decreased apoptosis, and local disturbances in the levels of normal lung growth factors have been suggested as mechanisms underlying CCAM (4). Imbalance in the blood flow in the recipient with TTTS, causing local disturbances in the levels of normal lung growth factors may play an important role in the development of CCAM in the recipient twin. MRI can serve as a helpful and complementary tool to US for the detection of complications in fetuses with TTTS.

\section{Footnote}

Authors' Contributions: Yu-Peng Liu, first author and corresponding author; Yang-Kai Fan and Yi-Lan Lin, MRI acquisition; Chih-Ping Chen, case collection; Wen-Ko Su, revision of the manuscript and final approval.

\section{References}

1. Kline-Fath BM, Calvo-Garcia MA, O'Hara SM, Crombleholme TM, Racadio JM. Twin-twin transfusion syndrome: cerebral ischemia is not the only fetal MR imaging finding. Pediatr Radiol. 2007;37(1):47-56. doi: 10.1007/s00247-006-0337-5. [PubMed: 17063351].

2. Liu YP, Chang TY. Congenital cystic adenomatoid malformation in twin-twin transfusion syndrome: fetal MR imaging. Pediatr Radiol. 2007;37(9):941. doi: 10.1007/s00247-007-0530-1. [PubMed: 17593361].

3. Quintero RA, Morales WJ, Allen MH, Bornick PW, Johnson PK, Kruger M. Staging of twin-twin transfusion syndrome. J Perinatol. 1999;19(8 Pt 1):550-5. [PubMed: 10645517].

4. Fromont-Hankard G, Philippe-Chomette P, Delezoide AL, Nessmann C, Aigrain Y, Peuchmaur M. Glial cell-derived neurotrophic factor expression in normal human lung and congenital cystic adenomatoid malformation. Arch Pathol Lab Med. 2002;126(4):432-6. [PubMed: $11900567]$. 